\title{
The research and development of energy consumption detection and analysis management systems based on the digital slurry pipeline
}

\author{
Hongwei Liu ${ }^{1, a}$, Xingxing Yao, ${ }^{2, b}$ and Chunlei Pan $^{3, c}$ \\ 1Yunnan Da Hongshan Pipeline Co., Ltd., Kunming, 650504, China \\ 2Faculty of Information Engineering and Automation, Kunming University of Science and \\ Technology, Kunming, 650500, China \\ a1162494119@qq.com, b530703461@qq.com, ${ }^{\text {c }}$ steelkm@foxmail.com
}

\begin{abstract}
Keywords: Energy consumption detection and analysis; energy consumption; Information management system; Silverlight
\end{abstract}

\begin{abstract}
Detection and analysis of energy consumption is the information management systems, which not only help producers to expand production, but also rationally plan and use the energy, reduce energy consumption per unit of product, improve economic efficiency. The company managers can have an accurate grasp for the proportion of energy costs and the development trend of enterprises by energy plan, energy monitoring, energy statistics, energy consumption analysis, important energy equipments management, energy metering equipments management and other means. The enterprise energy consumption plan tasks are decomposed to each production sector workshops, which makes energy conservation and responsibilities clear and promotes the healthy and stable development. This paper takes slurry pipeline as background, combining with Silverlight software platform to design the energy detection and analysis management systems based on the digital slurry pipeline. The actual application shows the effectiveness and versatility of the program.
\end{abstract}

\section{Introduction}

Dahongshan Pipeline Company is the services companies, which is specialized in the slurry pipeline transportation and other solid material transportation. The pipe line of Dahongshan pipeline has a steep terrain and a total length of $171 \mathrm{~km}$ so as to become the first length iron ore transportation in the national. In the development process, Dahongshan Pipeline Company has already built the website platform, integrated management and control systems, data acquisition and monitoring SCADA systems, slurry characteristics management system and video surveillance and other information platforms. However, due to the detection and analysis of energy in Dahongshan pipeline decentralized, a single management tools and other reasons so that Dahongshan Pipeline Company has not established a rational and scientific energy detection and analysis management systems.

Digital slurry pipeline is a strategic, which can support business development. In the management philosophy of "unified planning, step by step, the key breakthrough", advocating and supporting the digital management for production, real-time monitoring for the production site and dynamic analysis and decision for production data, which can ensure enterprises to get better development in the secure and stable environment. This article is the system solution based on a digital pipeline. According to the specification standard of the enterprise energy management, the system rationally allocates the department roles and characters by analyzing the key processes and important part of energy detection and analysis management so as to achieve the online management for energy detection and analysis management activities, which not only achieves a resource sharing of energy detection and analysis information, but also makes all related departments work together better . Moreover, it can enhance the economic efficiency of enterprises. 


\section{System design solution}

The process, device, the product types and the level of energy management will produce different effects to energy consumption in different types of industrial enterprises. Construction of a plant-wide centralized energy management system can carry out online collection, calculation, analysis and processing of energy data so as to play an important role in the energy materials balance, scheduling and optimization technology and operation and management of other energy equipment . The systems have established a management standards based on actual energy detection and analysis management, achieving clarity of workflow, arriving energy management and maintenance intelligent, etc.

Through a combination of the production and operation management standards in major companies, according to the implementation guidelines for energy detection and analysis management, energy detection and analysis management activities are divided into the following phases:

(1)Take unified specification、standard classification and management on basic information of energy.

(2) Take acquisition for energy consumption data.

(3) Take management and maintenance for energy metering equipment.

(4) Take effective monitoring for the relevant energy dynamics.

(5) Take statistic and analysis for energy consumption data.

The function structure diagram of energy detection and analysis management system is as follows:

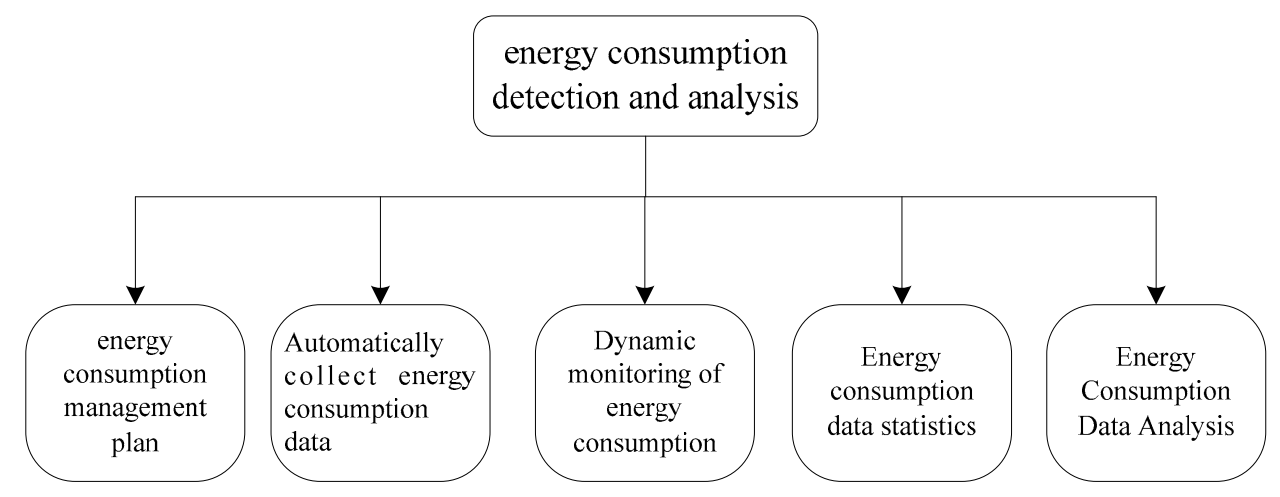

Fig.1 The function structure diagram of energy management system

\section{System workflow analysis}

Energy consumption management plan. Every work area reports annual energy consumption management plan in each annual, which can guide the energy consumption requirements for the year, at the end of year, compared energy consumption management plan with actual performance comparison to determine the completion of annual energy consumption. Secondly, the work area can develop the monthly energy consumption management plan according to annual energy consumption management plan and production tasks to guide the month energy consumption requirements, at the end of month, compared energy consumption management plan with actual performance comparison to determine the completion of monthly energy consumption. Meanwhile, the benchmarking mechanism can be established between pumping stations and pumping stations, work area and work area, the pipeline and the pipeline. By judging from the pump speed of downstream pipeline, flow rate, pressure, moisture and other process parameters, providing the advice of device switches to effectively conserve energy, reduce costs and optimize the acquisition time node of existing energy data so as to narrow differences in settlement data with the power companies and improve the existing circuit meter structure, thereby achieve energy effective management. 
Automatically collect energy consumption data. Automatic collection methods of collect data, including statistical breakdown data and classification energy consumption data, which can be took real-time acquisition by the automatic measuring device, real-time transporting to the data center or transfer station by automatic transmission. Data collection terminal of the workspace field automatically accomplishes energy consumption data collection in the system and then uploading energy consumption data by team.

Dynamic monitoring of energy consumption. The system allows to set the alarm thresholds of itemized energy consumption, the total energy consumption and unit consumption, when itemized energy consumption, total energy consumption and unit consumption exceed the alarm threshold, the system will take real-time alarm in striking position ${ }^{[2]}$; Supervising officer can also directly take tracking and monitoring for the target buildings, which makes energy management "move up". Digital pipeline SCADA system can real-time collect and monitor energy consumption data, real-time monitoring the condition parameters and operation situation of the intelligent devices within the system so as to intelligently control and maintain the related equipment.

Building energy consumption dynamic monitoring is to improve the management level of the digital pipeline, quickly and accurately obtaining energy consumption information of run-time, completely grasping the real-time operating status of the pipeline, detecting faults of the pipeline running and making the appropriate decisions and treatment while management staff on duty can take load analysis and reasonable dispatch according to the operation situation of the system, which can seize the initiative of security control and incident handling, reduce and avoid the operation misjudgment and achieve a modern operation management of energy consumption in digital pipeline system.

Energy consumption data statistics. Energy utilization can be took statistics by Classification statistics, statistical breakdown, fold standard statistical, energy consumption statistics and energy consumption costs statistics and so on, statistics periods can choose hourly reports, daily reports, monthly reports and annual reports ${ }^{[3]}$ so that the government regulators quickly and accurately grasp the energy information of each dimension and generate the required statistical reports.

Energy Consumption Data Analysis. On the basis of the energy consumption statistics, multi-dimensional comparison and analysis can be carried out by region, team, operating parameters, constants for the energy consumption monitoring areas, including trend analysis of energy consumption, energy consumption comparison in the same period, the sort of high energy consumption and so on. By beautiful graphical reports, status-quo facts of energy utilization can be clearly showed, which can help regulators quickly analyze the energy utilization trends in massive amounts of data, analysis the loopholes and shortcomings of management and form decision support system. The flow diagram of energy consumption data is shown in Fig.2.

\section{Summary}

This paper discusses the technical solutions to achieve application framework of energy detection and analysis management system based on the digital slurry pipeline. This application framework has been successfully used in the design and development practices of energy detection and analysis management system in Dahongshan digital pipeline platform, its application core module has been developed, at present, the system is putting into application and debugging in Dahongshan Pipeline Company. Practice shows that this system demonstrates the unique advantages, which has the following advantages: The standard work flow provided by the system provides a convenient to the relevant department heads, reduce the workload and improve work efficiency; Users can understand real time progress of the work flow by the system. The network share of energy information enhances the coordination of various departments. Energy detection and analysis is based on a statistical basis of actual production energy consumption, energy detection and analysis mainly makes reasonable, organic imputation and analysis for the energy produced by various departments and aspects in the actual manufacturing operations. In order to deal with energy detection and analysis well, it is necessary to communicate with Production Management, Process Technology and Engineering 
Center and other departments, based on the actual needs of energy detection and analysis management, ultimately establishing management standards, achieving clarity of the work flow, making sure energy detection and analysis management and intelligent maintenance, etc.

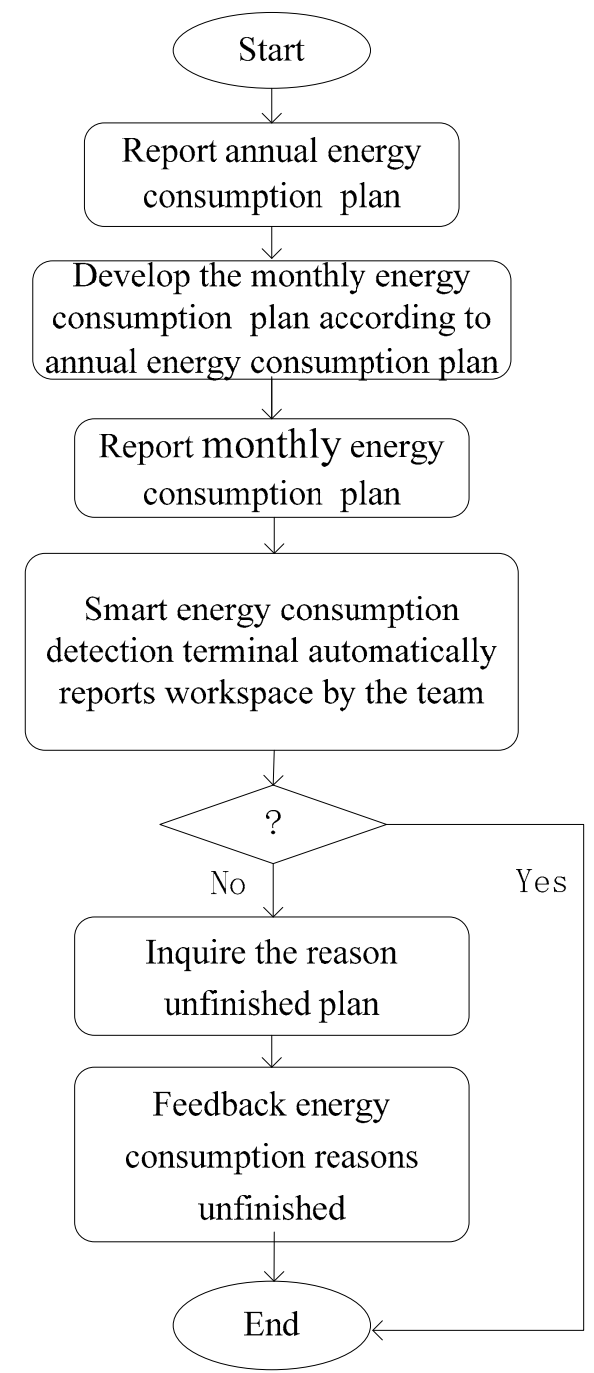

Fig.2 The flow diagram of energy consumption data analyze

\section{Acknowledgement}

This work is supported by National Natural Science Foundation of China (No. 51169007), Science \& Research Program of Yunnan province (No.2011CI017 \& 2012CA022\&2013DH034).

\section{References:}

[1] Fu Wei, Fang Jian.Design of Energy Consumption Detector Based on Wireless Sensor Network [J]. Automation Instrument,2013,34(7):49-53.

[2] Tompros S,Mouratidis N,Draaijer M,et al.Enabling application of energy saving appliances of the home environment [J].IEEE Network, 2009,23(6):8 - 16 .

[3] Li CH,Bai YW,Lin MB,et al.Remote-controllable power outlet system for home power management[J].IEEE Transactions on Consumer Electronics， 2007， 53(4):1634 - 1641. 
[4] YANG Huiliang, SUN Yining, LI Dan,ZHANG Xu. Design and Develop of Human Body Energy Consumption Detect System Based on Android Mobile Phone Platform[J].Instrumentation Technology,2013,(7):13-16.

[5] ZHU Guozhong,WEI Caihong,PAN Min.The Research of Energy Expenditure Detection Algorithm Based on Tri-Axial Acceleration Transducer. Chinese Journal of Sensors and Actuators, 2011, 24(8): 1217-1222. 